\title{
Signal-detection experiments as tests for risk-taking: A pilot study
}

\section{Y. H. POORTINGA, ${ }^{1}$ National Institute for Personnel Research, Johannesburg, South Africa}

Auditory and visual signal-detection experiments were applied as tests of risk-taking. The percentage of errors in the responses was kept constant over Ss and blocks of trials by slight variations in signal strength. Ss could express two levels of confidence for their decisions. The numbers of more confident decisions in the two experiments are highly correlated, especially under conditions of small monetary risk.

This investigation is part of a larger project to develop tests consisting of simple stimuli and responses which do not demand culturally specific knowledge or experience and are, therefore, applicable in a wide range of cultures (Poortinga, 1967). Signal-detection experiments are simple and seem to be suitable for the study of certain aspects of decision-making.

The experimental techniques are based on Signal Detection theory (Green \& Swets, 1966). The intensity of the "signal" was manipulated by the $E$ so that the percentage of errors was held more or less constant over blocks of trials and over Ss. It is assumed that in this way the effects of differences in sensitivity between individuals and changes within individuals over time are cancelled out and that only the "criteria" used by the Ss determine differences in responses. Ss gave a confidence rating (more sure or less sure) for each answer. Risk-taking was emphasized by linking the rating to monetary rewards and losses. For a less confident answer the amount which could be earned or lost was smaller (or nil) than for a more confident response. The aim of this investigation was to establish whether Ss who had no previous experience in the type of task would show consistent differences in risk-taking over a relatively short period of testing. Two parallel experiments (visual and auditory) were administered to check whether the results were reliable and independent of the type of stimulation.

\section{METHOD}

In the auditory experiment the "noise" was formed by white noise of approximately 60-dB intensity, while the signal was a pure tone of $1000 \mathrm{~Hz}$, with continuously variable intensity. Both were generated by a B 222 ET Pedersen audiometer and presented to the left ear of the Ss by means of the audiometer's headphones. The presentation of the signal was controlled by an on-off switch. The visual experiment was carried out in a dimly illuminated room; the illumination was switched off for the duration of each stimulus. Two identical quartz-iodine lamps were used. The light of the one was filtered with a neutral density filter and served as noise. The light of the other passed through an iris-diaphragm by means of which the intensity could be varied, and through a Kodak Wratten filter (No. 70) to produce a deep red signal. Light from both sources was projected onto a piece of white translucent perspex (1.5-in. diam); the $S$ looked at this from the other side. The presentation of the signal was controlled by means of a silent shutter.

In these experiments, unlike most previous investigations, noise (like the signal) was presented only for the duration of the stimulus time. This made it possible for $E$ to control the presence or absence of the signal with the hand-operated switch or shutter.

\section{PROCEDURE}

Stimuli were presented in blocks of 100 . The percentage of errors was kept as close to $25 \%$ as possible by slight manipulations of the signal intensity, when $\mathrm{E}$ observed that the number of errors relative to the number of correct responses became too large or too small. At the beginning of a session the signal was shown a few times without the noise. The signal was presented in 50\% of the trials (randomly selected). Each trial lasted $8-1 / 2 \mathrm{sec}$ and consisted of the following: warning signal $(1 / 2 \mathrm{sec})$; interval $(1 / 4 \mathrm{sec})$; stimulus presentation $(2-1 / 8 \mathrm{sec})$; response and feedback $(5-5 / 8 \mathrm{sec})$.

The Ss indicated their decision as to whether the signal was present or absent by pressing one of four buttons (the outer two indicating "more confidence," the inner two indicating "less confidence"). When Ss made an error they were immediately informed about it.

\section{SUBJECTS}

Twenty male Africans, undergraduate students of the University College of Zululand varying in age between 21 and 36 years, participated as Ss. They were prescreened for acuity of hearing and color-blindness. Eighteen of them completed both experiments. They were paid for their cooperation, the amount being partly dependent on their performance.

\section{EXPERIMENT AL GROUP 1}

The first group of nine students was given a considerable amount of practice. During three sessions a total of 1100 stimuli per experiment was presented. The order of the experiments within the sessions was systematically varied. In the first session an introduction and three blocks of trials for each experiment were given; in the second and third sessions four blocks of trials per experiment were given.

To prevent the establishment of fixed strategies during the training periods, Ss were asked to give more or less the same number of answers in each of the four response categories. To facilitate this they were given four trays with 25 chips each, at the start of every block of stimuli. Each time a button was pressed a chip had to be moved from the corresponding tray.

Two measurements of risk-taking were obtained, one without and one with a monetary reward system. For the last two blocks of the second session the Ss were instructed to consider a more confident response as better than a less confident one if correct, and as worse if wrong. For the last two blocks of the last session Ss were promised 1c $\quad=$ Rand 0.01 ) per more confident and $1 / 2 \mathrm{c}$ per less confident correct response, against a loss of $3 c$ and $11 / 2 c$ for a wrong response. Since Ss were correct approximately $75 \%$ of the time the expected earnings in the case of random answering or a fixed strategy were nil.

\section{EXPERIMENTAL GROUP 2}

The second group of nine students was given only four blocks of stimuli per experiment in two separate sessions. The auditory experiment was always administered first. During the training (first two blocks) Ss decided for each trial only whether the signal had been presented or not (using the two buttons in the middle). The use of chips to elicit an equal number of answers in each category was discarded. Risk-taking was measured over the last two blocks. The Ss were instructed to press one of the extreme buttons when they felt sure enough about their response to stake $3 \mathrm{c}$ for a possible gain of 1c. (Pressing of the middle buttons did not have monetary consequences.) For this group payments in the form of chips were made after each response (if applicable) so that Ss could keep track of their earnings.

\section{RESULTS}

The results obtained from the first group during the training deviated from the equal number of answers expected in each 
class on the basis of the instruction. In the visual experiment the distribution of the number of responses given in each class (the classes are of course negatively correlated) yielded an $\mathrm{SD}=10.1$ and an $\mathrm{SD}=7.8$ for the first half of the second and third sessions, respectively. For the auditory experiment SD values of 9.6 and 12.6 were found. It was concluded that the use of the trays with chips was not entirely satisfactory, but since most $\mathrm{Ss}$ did not show a fixed strategy (the one $\mathrm{S}$ who did so in both experiments had no fixed strategy during the training) the results were analyzed further.

The intra-individual changes over time in the intensity level at which Ss made $25 \%$ errors were in both groups considerably smaller than the range of the means of intensity levels over individuals. ${ }^{2}$ The rank correlations between these means and the number of more confident responses were low so that differences in the latter could not be explained in terms of differences in signal intensity between Ss. The values for the first group were $r_{s}=0.02$ in the visual experiment and $r_{s}=0.14$ in the auditory experiment for risk-taking with money; $r_{s}=0.14$ and $r_{s}=0.12$ for risk-taking without money. The values for the second group were $r_{s}=0.42$ and $r_{s}=0.19$, respectively.

The $E$ could usually keep the number of mistakes fairly close to the required 25 per block (cf. Table 1 ).

The number of times a more confident response was given over 200 trials has been taken as the risk-taking score. The intercorrelations for the first group between the two conditions (with and without money) are given in Tables 2 and 3.

The intercorrelation between the scores in the second group was $r=0.84$. If the two experiments in each group are looked upon as parallel-tests, the reliability appears to be acceptable at least under conditions of monetary risk and the results seem to be quite independent of the type of stimuli applied. This is in agreement with other findings (Carterette \& Cole, 1962).

In Fig. 1 the scores obtained in the two groups under the condition of monetary reward are given. ${ }^{3}$ The amount of money earned is a linear function of the distance of each score from the diagonal. The lack of a clear relationship between these distances and the number of confident responses implies
Table 1

Means and SDs of the Distributions of Number of Mistakes

\begin{tabular}{crrrrrr}
\hline & \multicolumn{4}{c}{ Group 1 } & \multicolumn{2}{c}{ Group 2 } \\
\cline { 2 - 6 } & \multicolumn{3}{c}{ With money } & \multicolumn{3}{c}{ Without money } \\
\cline { 2 - 7 } & Visual & Auditory & Visual & Auditory & Visual & Auditory \\
\hline Mean & 53.8 & 51.0 & 50.3 & 51.3 & 52.3 & 52.8 \\
SD & 4.7 & 2.2 & 3.2 & 3.6 & 3.0 & 3.6 \\
\hline
\end{tabular}

Table 2

Inter-Correlations Between Visual and Auditory Experiments in the First Group

Auditory without money

Visual without money

Auditory with money

Visual with money

.61

$.47 \quad .36$

$\begin{array}{lll}.68 & .63 & .90\end{array}$

Table 3

Inter-Correlations Between First and Last Administered Experiments in the First Group

First Experiment without money

Second Experiment without money

First Experiment with money

Second Experiment with money

.67

$.65 \quad .55$

$\begin{array}{lll}.49 & .65 & .89\end{array}$

that the results in general did not favor a change in decision strategy during the experiment. In spite of the performance of two $\mathrm{Ss}^{4}$ in the first group, on the visual experiment, it seems that even largely untrained Ss can differentiate between less confident and more confident judgments in a nonrandom fashion. The tasks can therefore be considered to be meaningful.

In conclusion, it appears that the experiments differentiate reliably between $\mathrm{Ss}$ in terms of the relative frequency of more confident responses. In view of the experimental framework, these results cannot readily be explained in terms of some perceptual difference, and it therefore seems likely that risk-taking is indeed being measured. ${ }^{5}$

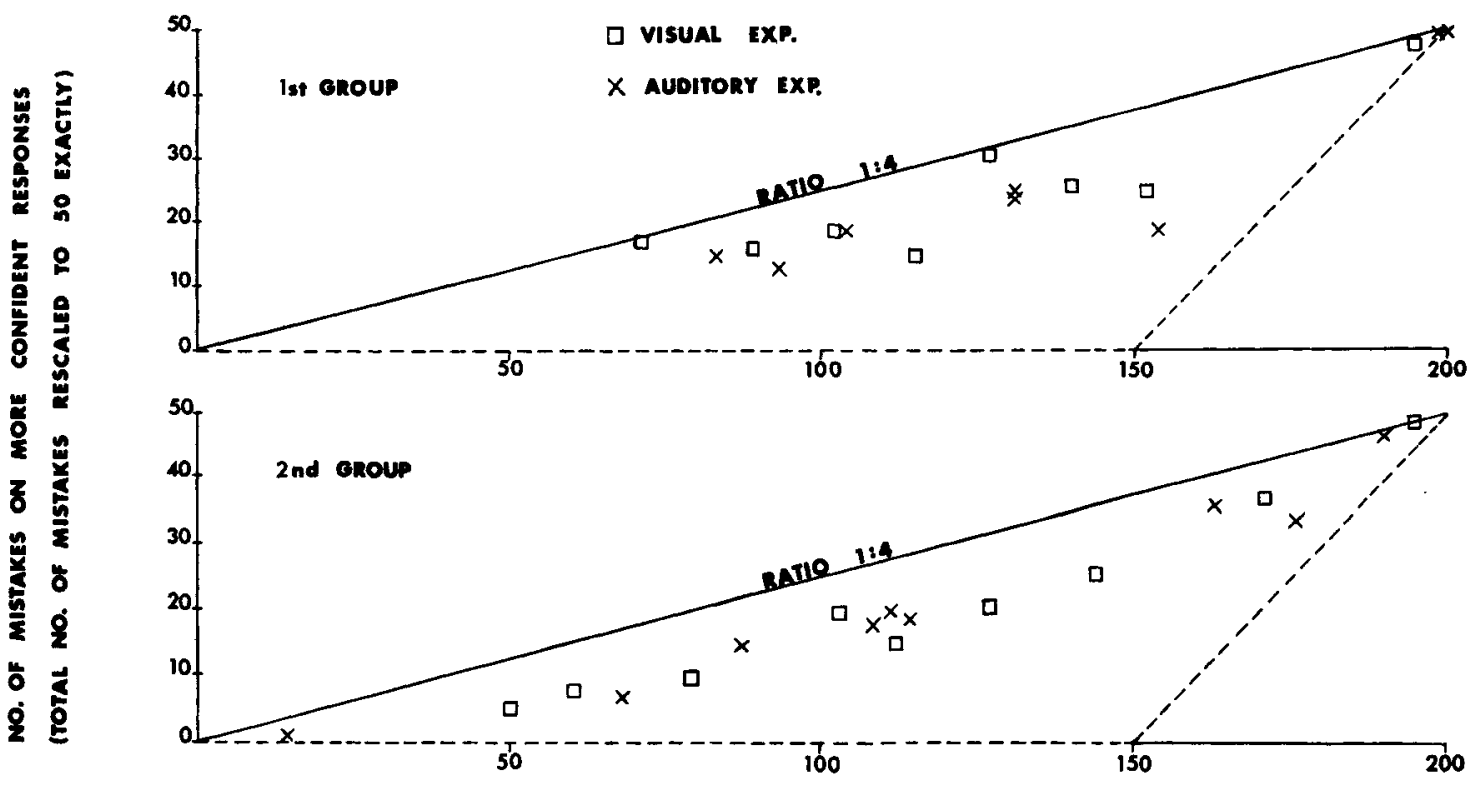

NO. OF MONE CONFIDENT RESPONSES

Fig. 1. Number of incorrect more-confident responses over the total number of more-confident responses for each $S$. The dotted line indicates the maximum distance of scores from the diagonal.

(Continued on page 188) 


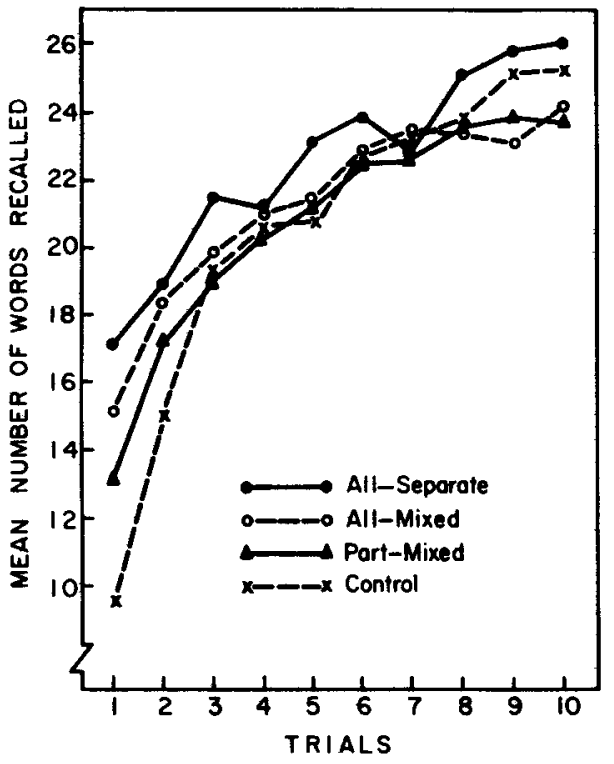

Fig. 2. The mean number of words correctly recalled for the $\mathbf{1 0}$ trials on List 3 for the four conditions of Experiment 2.

same presentation orders of List 3; each S received three different orders of his three lists. Except for the restrictions imposed by the design, the presentation orders were random. The words were taken from Deese's (1959) zero interitem associative strength lists.

Subjects were run in groups. Lists 1 and 2 were presented in booklets with a new page for each of the five trials. Ss had 1 sec per word to read each list and $\mathbf{4 5} \mathrm{sec}$ for written recall. At the end of each presentation, Ss closed their study booklets and wrote the items they could recall in their answer booklets. The final list was presented by a slide projector for 10 alternating study and test trials. The words were presented at the rate of one word every $2 \mathrm{sec}$ with $90 \mathrm{sec}$ for written recall.

\section{Results and Discussion}

Performance on Lists 1 and 2 was analyzed to determine whether the groups differed prior to List 3 learning. Each S's score consisted of the number of words correctly recalled on Trial 5 of both lists. The means for the four groups, which ranged from 22.78 to 24.17 , were not significantly different $(\mathrm{F}<1)$.

The mean number of words correctly recalled for List 3 is presented in Fig. 2. There was no difference as a function of Conditions ( $F=1.66, \mathrm{df}=3 / 68, p>.05)$, but the slopes of the curves differed for the four conditions $(F=71.05$, $\mathrm{d} f=3 / 612$, $\mathrm{p}<.001)$. The Part-Mixed and Control groups were essentially identical to the conditions in Tulving's (1966) study except that a group presentation procedure was used in the present study. A separate comparison of these groups revealed that the slopes of the curves differed when all 10 trials were considered $(\mathrm{F}=156.00, \mathrm{df}=1 / 612, \mathrm{p}<.001)$, but not when Trials $4-10$ were considered $(\mathrm{F}=3.58, \mathrm{df}=1 / 612, \mathrm{p}<.10)$. It appears that the difference in slopes was largely a result of the initial positive transfer for the experimental Ss. However, the fact that the positive transfer for experimental Ss was not maintained can be viewed as support for Tulving's notion that higher-order memory units formed during List 2 learning interfered with List 3 learning.

A comparison of the All-Mixed group and the Control group was made to determine if the negative transfer resulting from prior practice with final list items would be obtained when all the items were practiced prior to final list learning. An analysis of the number correct for Trials 4-10 revealed that the slopes of the curves differed $(F=9.60, \mathrm{df}=1 / 612, \mathrm{p}<.01$ ). This finding is rather strong support for the view that prior experience with items is not necessarily helpful. An examination of Fig. 2 yields weak evidence to support the view that presentation order influenced performance. A comparison of the All-Separate and All-Mixed groups with the Control group revealed that negative transfer late in List 3 learning was obtained only when the List 1 and List 2 items were not presented consecutively for List 3.

Bousfield \& Bousfield's (1966) measure of stimulus category repetition (SCR) was employed to compare organization of recall on Trial 10 with previously learned organization from Lists 1 and 2. The Part-Mixed group had previous practice on odd or even final list items. Each previously learned list was viewed as a category to determine whether words presented together during List 1 and 2 learning would be recalled together on Trial 10 of List 3 learning. The difference between the obtained and expected value of SCR was computed for each S.

The mean SCR scores (obtained minus expected) based on odd and even List 3 categories were .97, 1.33, and -2.39 for the All-Mixed, Part-Mixed, and Control groups, respectively. An orthogonal comparison between the average of the mixed groups and the Control group was significant $(\mathrm{F}=23.44$, $\mathrm{df}=1 / 51$, $\mathrm{p}<.001$ ), indicating that the mixed groups maintained, at least in part, their prior organization of Lists 1 and 2 throughout final list learning. This result supports Tulving's contention that Ss may be unwilling or unable to abandon their previously learned organization.

\section{REFERENCES}

BOUSFIELD, A. K., \& BOUSFIELD, W. A. Measurement of clustering and of sequential constancies in repeated free recall. Psychological Reports, 1966, 19, 935-942.

DEESE, J. Influence of inter-item associative strength upon immediate free recall. Psychological Reports, 1959, 5, 305-312.

POSTMAN, L. Association and performance in the analysis of verbal learning. In T. R. Dixon and D. L. Horton (Eds.), Verbal behavior and general behavior theory. Englewood Cliffs, N. J.: Prentice-Hall, 1968. Pp. 551-571.

TULVING, E. The effect of order of presentation on learning of unrelated words. Psychonomic Science, 1965, 3, 337-338.

TULVING, E. Subjective organization and effects of repetition in multi-trial free-recall learning. Journal of Verbal Learning \& Verbal Behavior, 1966, 5, 193-197.

\section{NOTE}

1. This investigation was supported in part by Public Health Service Research Grant MH 14063. This manuscript is based in part on a thesis submitted by Dorothy Clark to Michigan State University in partial fulfillment for the degree of Master of Arts, November 21, 1967.

\section{REFERENCES}

CARTERETTE, E. C., \& COLE, M. Comparison of the receiver-operating characteristics for messages received by ear and by eye. Journal of the Acoustical Society of America, 1962, 34, 172-178.

GREEN, D. M., \& SWETS, J. A. Signal-detection theory and psychophysics. New York: Wiley, 1966.

POORTINGA, Y. H. The comparability of scores obtained in different cultures. Paper read at the Annual Congress of the South African Psychological Association, 1967.

\section{NOTES}

1. The author's stay at the Institute has been made possible by an Advanced Research Fellowship from the Anglo-American Corporation of

\section{(Continued from page 186)}

South Africa.

2. The range of means for the auditory experiment varied between approximately $42 \mathrm{~dB}$ and $48 \mathrm{~dB}$. The visual scale was not calibrated in terms of common standards.

3. The results of the first group under conditions of no monetary reward were very similar to those given in Fig. 1.

4. Most of the scores obtained by one of these Ss during the training also fell close to the diagonal.

5 . In order to confirm this, a number of other risk-taking tests were administered to all Ss. The intercorrelations between these and the signal detection experiments were mostly in the expected direction. They do, however, require confirmation with larger samples. 